\title{
Fusion and quasi-fission in the formation of heavy elements
}

\author{
D.J. Hinde ${ }^{\mathrm{a}}$, R du Rietz and M. Dasgupta \\ Department of Nuclear Physics, Research School of Physics and Engineering, \\ Australian National University, Canberra, ACT 0200, Australia
}

\begin{abstract}
The time scales of the dynamical nuclear rearrangement processes of fission following fusion, and of quasifission, are significant tests of models of nuclear dynamics. Experimental approaches to determine characteristic times, and their consistency, are discussed.
\end{abstract}

\section{Introduction}

Understanding the dynamical competition between quasifission and fusion will lead to more reliable predictions of opportunities to form a wider range of isotopes of superheavy elements (SHE) in nuclear fusion reactions. The presence of fusion-fission is a signature that fusion has occurred, and a compound nucleus $(\mathrm{CN})$ has been formed. If the $\mathrm{CN}$ evaporates only neutrons, this results in a heavy residual nucleus which is the desired SHE. Much more likely, the $\mathrm{CN}$ can split into two fragments, generally of similar mass, which is the familiar process of fission. Both premature breakup after capture - quasi-fission - and compound nucleus fission inhibit the formation of SHE. The latter is a property of the $\mathrm{CN}$, whereas the extent of quasifission competition can be changed by choice of target and projectile nucleus. Thus a thorough understanding of this processes is the key to realize opportunities to form more isotopes of SHE in the laboratory.

The characteristics of the products of fusion-fission and quasi-fission show considerable overlap. Thus it is difficult to unambiguously separate quasi-fission and fusionfission, though ingenious fitting procedures have been developed [1]. However, as noted, quasi-fission and fusionfission occur at different stages of evolution of the composite system, which is reflected in the difference in their average timescales. Quasi-fission has generally been understood [2] to occur on short time scales $\left(\leq 10^{-20} \mathrm{~s}\right)$. Fusionfission typically occurs on longer time scales, perhaps from as short as $\sim 10^{-20} \mathrm{~s}$, up to $\sim 10^{-16}$ s. Determination of fission times can thus give a clear signature of the dominant process. Three main methods have been used to infer time scales, each with a different sensitivity. The consistency of times extracted by these three methods will be addressed.

\section{The neutron clock}

The neutron-clock method [3] counts the neutrons emitted from thermalization to breakup into two fragments (scis-

\footnotetext{
a e-mail: david.hinde@anu.edu.au
}

sion). It can be sensitive in principle to time scales from $10^{-22}$ to $10^{-16} \mathrm{~s}$. However, optimal interpretation of the measurements requires a priori knowledge of the dynamics, as well as neutron evaporation lifetimes [3] from all shapes encountered.

Generally for fusion-fission, the number of neutrons in excess of statistical model predictions [4] has been used to determine an additional dynamical time, corresponding to the sum of the times to build up flux over the fission saddle point, and the time to move from saddle to scission [3]. The latter work made use of measured mean prescission neutron kinetic energies to help determine neutron lifetimes [5]. A subsequent re-interpretation [6] of the multiplicities alone, neglecting neutron kinetic energies, led to up to a 10 times increase (from $\sim 10^{-20} \mathrm{~s}$ to $\sim 10^{-19} \mathrm{~s}$ ) in deduced fission time scales for both the fusion-fission and quasi-fission reactions [6]. However, it was later independently confirmed [7] that the analysis giving the longer time scales [6] is indeed inconsistent with the experimental neutron kinetic energies, and thus the long time scales cannot be justified.

For quasi-fission, a wholly dynamical process, the neutrons should measure the total time from energy thermalization to scission. The extracted time depends on the assumptions made, in particular in reactions forming heavy elements, depending sensitively on correct modeling of emission during fragment acceleration after scission [8]. This was conclusively shown experimentally for quasi-fission reactions [3] by the observed strong dependence of the extracted pre-scission neutron multiplicities $\left(v_{\text {pre }}\right)$ on the fragment total kinetic energy (TKE). This was not seen for fusion-fission $[3,9]$. The observation means that (many of) the neutrons identified as pre-scission are actually emitted after the TKE is determined. For reactions forming heavy elements, particularly when quasi-fission is present, the fragment excitation energies can be sufficient for neutron lifetimes to be shorter than the acceleration time of the fragments $[3,8]$. If this is the case, post-scission emission can contribute the the experimental $v_{\text {pre }}$. This occurs for two reasons. Firstly, in reactions forming heavy nuclei, the energy gain from the fission saddle-point to scission 

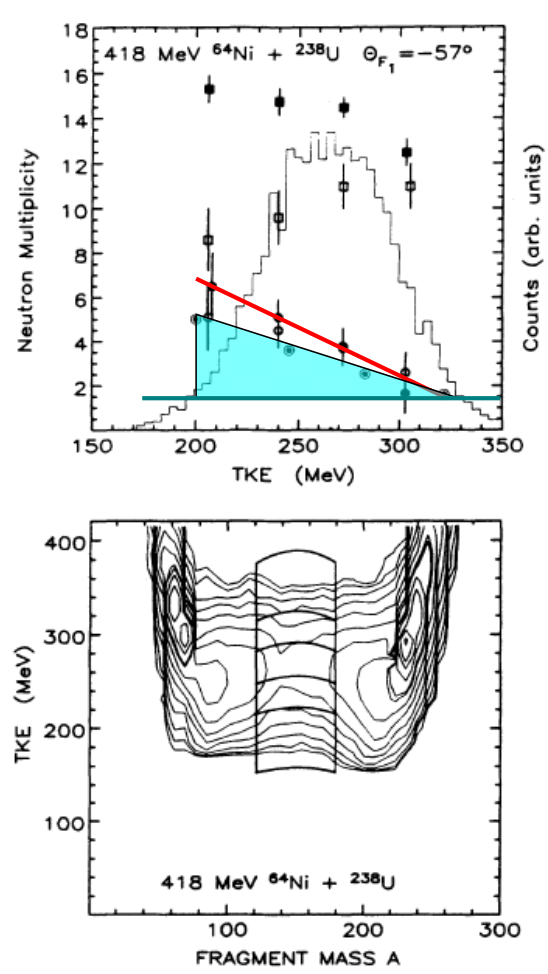

Fig. 1. Mass-energy distribution for the indicated reaction (below) showing gates applied to obtain the dependence of neutron multiplicities on total kinetic energy (TKE) shown above. Calculated apparent pre-scission multiplicities are given by the outlined circles, comprising a fixed true pre-scission yield (horizontal line) plus the acceleration neutron component (cyan shaded area). The best experimental values lie under the red trend-line. Open and closed squares show experimental post-scission and total neutron multiplicities.

can be larger than $50 \mathrm{MeV}$. Secondly, with short "sticking" times for quasi-fission resulting in fewer genuine prescission neutrons, the excitation energies at and after scission remain high. Neutrons evaporated after scission but prior to the fragments reaching close to their asymptotic velocity vectors will erroneously be substantially identified as pre-scission in the fits [8]. The number of these "spurious" pre-scission neutrons will depend sensitively on the TKE, since high TKE will result in low excitation energy, and thus fewer acceleration neutrons, whilst low TKE corresponds to high excitation energies.

To quantify this effect, a calculation was carried out [3] to estimate the number of neutrons emitted during fragment acceleration for the reaction $418 \mathrm{MeV}{ }^{64} \mathrm{Ni}+{ }^{238} \mathrm{U}$. The trend of the experimental $v_{\text {pre }}$ values is shown in Fig. 1 by the red line. Assuming a true pre-scission multiplicity independent of TKE, given by the horizontal line, the calculated contribution of acceleration neutrons to $v_{\text {pre }}$ is given by the cyan shaded region, resulting in a total $v_{\text {pre }}$ close to experiment.

As an independent test of the significance of acceleration neutrons, specific sources corresponding to the two accelerating fragments were later included in the fitting of the neutron angular correlations. The neutron detector angles
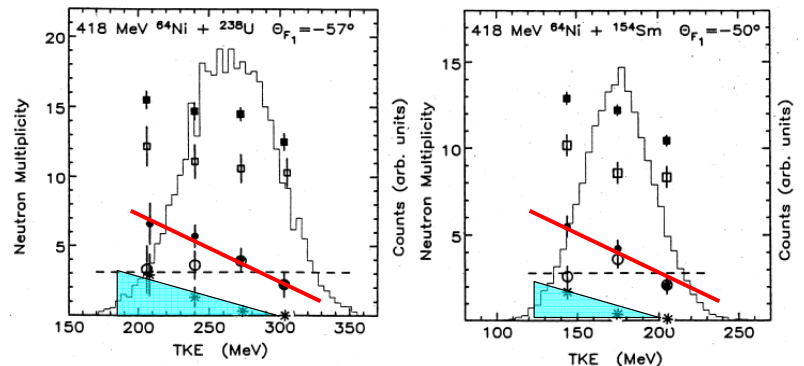

Fig. 2. As Fig.1, but including two accelerating fragments in the fits. The stars and cyan triangles indicate the apparent prescission multiplicities associated with the accelerating fragments. The open circles and dashed lines shows the pre-scission multiplicities and their average behaviour from the fits including acceleration neutrons.

were not chosen to be sensitive to such components in the angular correlation. Nevertheless, for the low TKE values, a significant improvement in fit quality resulted from inclusion of these sources. The multiplicities resulting from this fitting process for ${ }^{64} \mathrm{Ni}+{ }^{238} \mathrm{U}$ are shown in Fig.2. The original $v_{\text {pre }}$ (black circles and red trend line) are reduced (open circles), particularly at low TKE. The reduction in $v_{\text {pre }}$ due to including accelerating sources is indicated by the star symbols in Fig.2, whose average trend is represented by the cyan shaded triangle. Fit results consistent with those for ${ }^{64} \mathrm{Ni}+{ }^{238} \mathrm{U}$ were also obtained for the reactions of $418 \mathrm{MeV}^{64} \mathrm{Ni}$ with ${ }^{197} \mathrm{Au},{ }^{175} \mathrm{Lu}$, and ${ }^{154} \mathrm{Sm}$ (the latter being shown in Fig.2).

It was already concluded on the basis of calculated neutron lifetimes [3] that the neutron multiplicity identified as pre-scission in a three thermal source fit (C.N. and two fission fragments) to neutron angular correlations is an upper limit for those reactions where neutron emission during acceleration is significant. This is likely to include all quasi-fission reactions. The new evidence presented from fitting with accelerating fission fragment sources provides additional (experimental) evidence that this conclusion is correct. Thus quasi-fission times extracted from $v_{\text {pre }}$ values obtained from a three-source fit are almost certainly overestimates of the true pre-scission times.

Dynamical times (transient delay plus saddle-to-scission for fusion-fission, or sticking times for quasi-fission) determined [3] from $v_{\text {pre }}$ values extracted from three-source fits are shown in Fig.3. Reactions identified as having substantial quasi-fission contributions are marked with a downwardpointing arrow-head, indicating that the true quasi-fission times can be substantially smaller than those plotted. For reference, typical full rotation times [3] are indicated by the red curve. The consistency of these times with those extracted from Mass-Angle Distributions (MAD) will be discussed in Sect. 5.

Two further points are noted, arising from the preceding discussion of neutron emission during fragment acceleration.

The first is associated with fitting including accelerating fragments. The fits should require that the physics of sequential neutron evaporation and fission fragment veloc- 


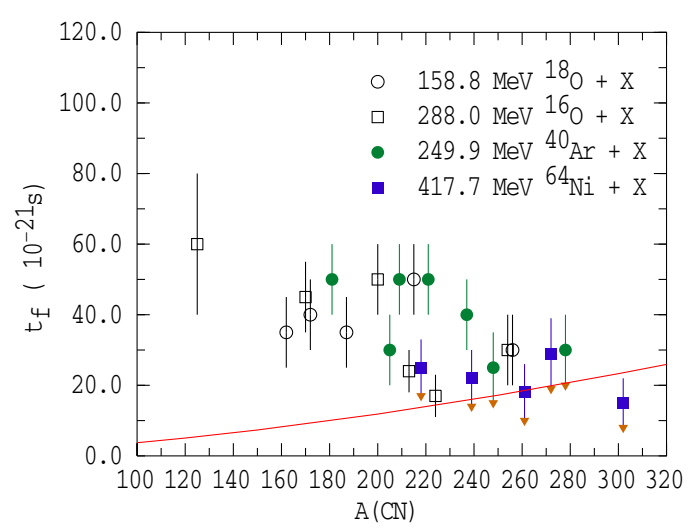

Fig. 3. Dynamical fission timescales from Ref. [3], as a function of compound nucleus mass number. Downward-pointing arrowheads indicate identified quasi-fission reactions, whose extracted time scales will in reality be shorter due to neutron emission during fragment acceleration. The red curve shows the variation of the typical full rotation time [3] with mass.

ities are linked through energy and momentum conservation. This takes the fitting process away from the simple physics of a three source fit, and moves it towards a dynamical model. Although a reasonable fitting procedure and outcomes were achieved as described above, it is our belief that once the fitting variables are linked in complex ways (as brought out in analysing the dependence of $v_{\text {pre }}$ on TKE $[3,9]$ ), a better approach should be to calculate the neutron angular correlation with a fully dynamical model, and change physically reasonable model parameters to obtain sophisticated information from complex experiments.

The second relates to the complex backtracing analysis $[10,11]$ of neutron angular correlations, a fitting method to extract not only average $v_{\text {pre }}$ values but also distributions of $v_{\text {pre }}$. This method will also be significantly affected by emission during acceleration for quasi-fission reactions.

\section{Mass-angle distributions}

The time scales of certain quasi-fission reactions have been determined from the rotation angle of the system before breakup [2,12]. This method can provide an almost modelindependent estimate of time scales. This is true only for some quasi-fission reactions, since the method is generally sensitive to timescales of $\leq 10^{-20} \mathrm{~s}$. This is because if the system makes more than one rotation, the fragment mass will no longer show a clear correlation with angle.

The relationship of the MAD to the lifetime of the system before scission is illustrated schematically in Fig. 4. The projectile nucleus (blue) is incident from above, and sticks to the larger target nucleus (red). The system then rotates, Fig. 4(b) showing angle against time (in arbitrary units) for a single angular momentum value - in a real reaction a distribution will be present. For a parabolic potential, mass-symmetry is approached with an expected time dependence $1-\exp \left(\tau / \tau_{e q}\right)$, where $\tau_{e q}$ is the mass-equilibration time constant [2]. This dependence is sketched in Fig. 4(c).

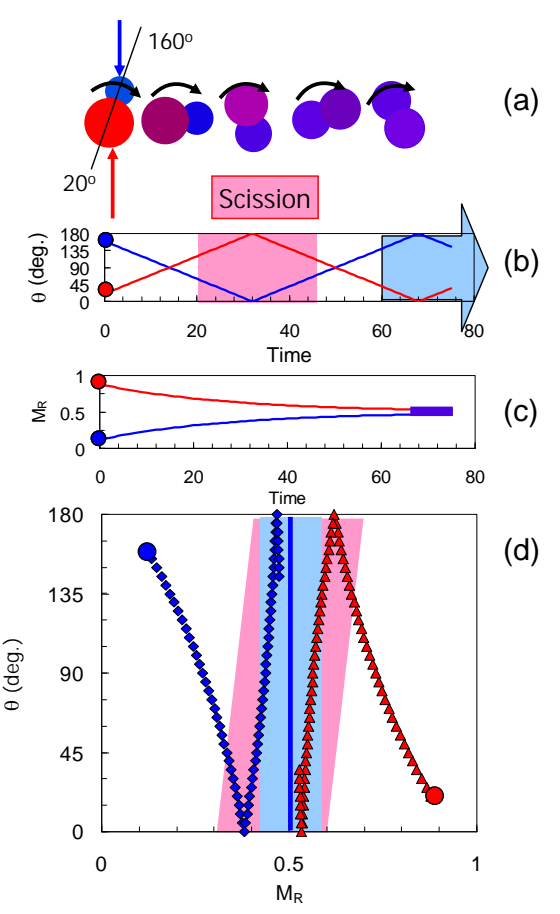

Fig. 4. Qualitative illustration of the relationship between reaction time and mass-angle distribution. For the time sequence of shapes sketched above, the time dependence (arbitrary units) of angle and mass-ratio are graphed below. Time is not directly measurable, but angle and mass-ratio are, the individual mass and angle dependencies combining to give a trajectory on the MAD (lower panel) for a single impact parameter. Including a range of impact parameters, scission after half a turn (pink) or a full turn (blue) will tend to give strong or weak mass-angle correlations respectively.

If scission occurs very soon after initial contact little mass change occurs, and a projectile-like fragment is ejected with mass-ratio $M_{R}$ at backward angle $\theta_{c . m .}$, its complementary partner with mass-ratio $\left(1-M_{R}\right)$ being seen at $\left(\pi-\theta_{c . m .}\right)$, a forward angle. An increase of the sticking time of the system results in larger rotation angles and more mass exchange. This evolution is illustrated on the MAD shown in Fig. 4(d). Rotation of the system by $\sim 180^{\circ}$ still results in a substantial mass-angle correlation (pink shading in Fig. 4(b),(d)), whilst once the system has turned $\sim 360^{\circ}$ or more, the correlation between the mass ratio and fragment emission angle is washed out, resulting in symmetric mass splits on average, independent of angle (blue shading).

Typical MAD measured at ANU are shown in Fig. 5. The experimental methods and analysis procedures are described in Refs. [13,14]. Examples for a fusion-fission reaction (left panel of Fig. 5) and a quasi-fission reaction (right panel) are shown. In the former case, symmetry about $\theta_{c . m .}=90^{\circ}$ is observed for all $M_{R}$, whilst in the latter case this is only true for $M_{R}=0.5$. Comparing with Fig. 4, the observed correlation of mass with angle is consistent with rotation angles of $\sim 180^{\circ}$. The corresponding time scale will be discussed in Sect. 5 . 

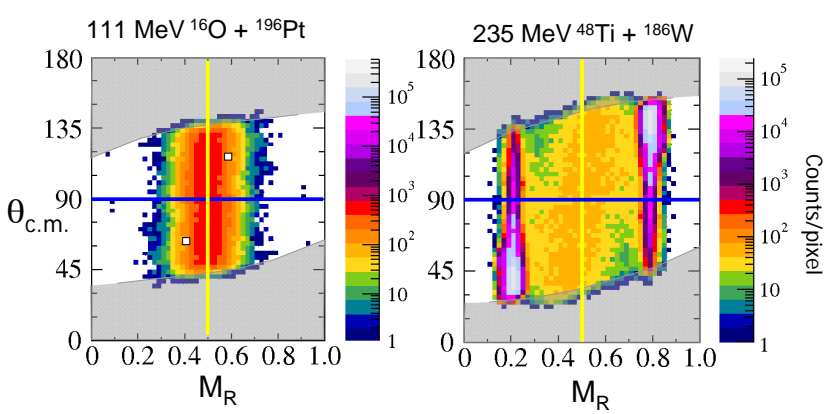

Fig. 5. Typical MAD for fusion-fission (left panel) and quasifission (right panel). The white squares represent the complementary points on the MAD populated by the detection in the experiment of two fission fragments in a single binary event. The grey shaded areas represent the regions of the MAD which could not be measured due to the limits of the detector angular coverage.

MAD which we have measured at the ANU show a consistent behaviour as a function of projectile and target mass/charge. This is illustrated in Fig. 6, where MAD are shown for a range of target nuclei on the horizontal axis, and projectile nuclei in the vertical direction. For ${ }^{16} \mathrm{O}$, there is no clear correlation of mass with angle, indicating no fission events occurring before a full rotation. The same is true for the ${ }^{32,34} \mathrm{~S}$ reactions, up to the ${ }^{232} \mathrm{Th}$ target, at which point a clear mass-angle correlation is visible, suggesting less than a full rotation before scission [15]. The shapes of the MAD remain similar for the reactions with the ${ }^{48} \mathrm{Ti}$ projectile, showing a very broad distribution of mass-splits [16], which, however, are still peaked at symmetry up to the ${ }^{238} \mathrm{U}$ target. Here the yield shows a minimum at symmetry, indicating a transition to still shorter

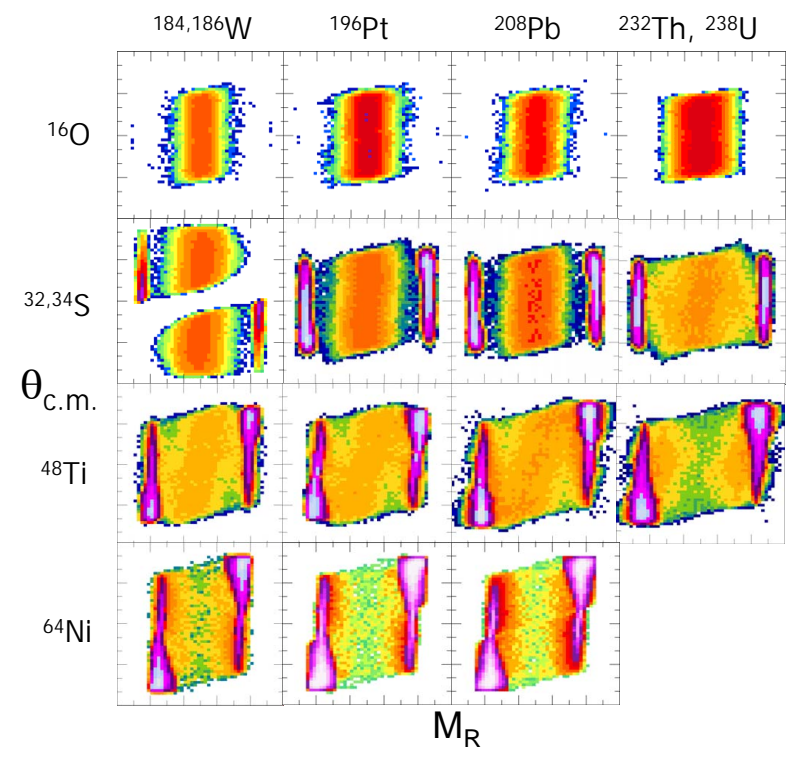

Fig. 6. Systematic variation of MAD as a function of projectile and target nucleus, at energies around $15-30 \mathrm{MeV}$ above the barrier. timescales. This character remains for the reactions of ${ }^{64} \mathrm{Ni}$, showing little change between ${ }^{184} \mathrm{~W}$ and ${ }^{208} \mathrm{~Pb}$.

These examples show that experimental MAD clearly show very different characteristics. The observed trends are generally as would be expected, both in terms of the competition between fusion and quasi-fission, and in the character of the quasi-fission itself. These expectations arise from general considerations of the Coulomb energy in the entrance channel, and the fissility of the compound nucleus [2].

\section{Crystal blocking}

The crystal blocking method measures the angular distribution of fission fragments [17] with respect to a major crystal axis of a crystalline target material. Fragments emitted in the direction of this axis are deflected away (blocked) by the row of atoms, unless the system has recoiled far enough from the lattice site (where it was formed) before scission occurs. This is sketched in Fig. 7. Although this method is insensitive to short times, it gives access to longer time scales, in the range $10^{-18} \mathrm{~s}-10^{-16} \mathrm{~s}$ [18]. It is thus sensitive to time scales associated with fusion-fission, and in principle can be used to indicate the presence of fusionfission events [19] amongst a strong background of quasifission events.

Recent measurements of crystal blocking for fissionlike events in reactions forming heavy elements have shown substantial filling of the blocking dips expected and observed for prompt reaction processes such as quasi-elastic and deep-inelastic $[18,19]$. This has been used to infer the presence of a substantial fraction of events with long fission times, in reactions where quasi-fission might have been expected to be the predominant process.

Blocking measurements were reported in Ref. [18,20] for beams of ${ }^{32} \mathrm{~S},{ }^{48} \mathrm{Ti},{ }^{58} \mathrm{Ni}$ and ${ }^{74} \mathrm{Ge}$, at energies per nucleon $\mathrm{E} / \mathrm{A}$ between 5.0 and $6.5 \mathrm{MeV}$, incident on $\mathrm{a}^{n a t} \mathrm{~W}$ crystalline target. These reactions form composite systems with atomic number $Z=90,96,102$ and 106 respectively. For the three heavier projectiles, the filling of the blocking dips increased with Z. It was concluded that for the selected fission-like events: (i) the measurements were consistent with a fission time distribution characterised by a

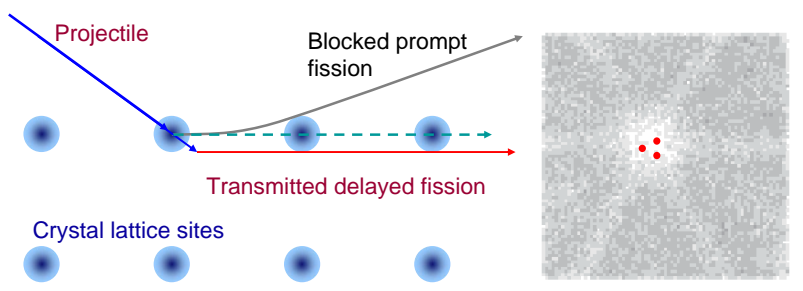

Fig. 7. Schematic diagram of the principle behind the crystal blocking technique. Prompt fission events are blocked along the line of the crystal axis. If the fission occurs after the compound nucleus has recoiled far enough from the axis, the blocking dip will be filled (red points on the blocking pattern at the right). 

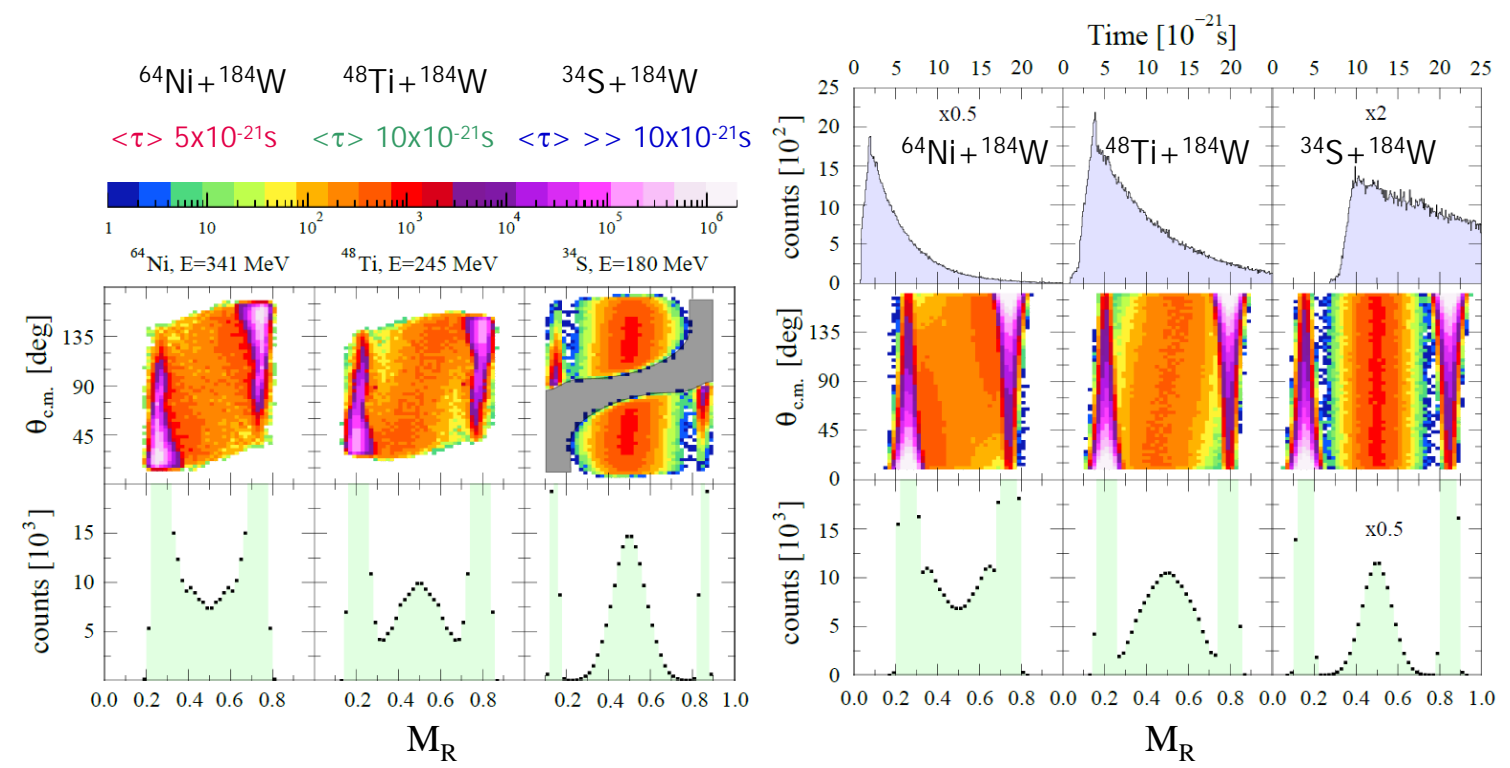

Fig. 8. (Left panels) Experimental MAD for the reactions indicated, with the projected mass-ratio spectra below. They show very different behaviour for each reaction. (Right panels) Simulated MAD which reproduce the experimental MAD. The time distributions needed to match the experiments are shown above the simulations. The deduced mean reaction times for each measurement (shown at the top left) are quite different, as expected from the very different MAD.

single exponential lifetime; (ii) all reactions have similar (long) mean lifetimes of just over $10^{-18}$; ; (iii) these times are truly characteristic of the processes giving rise to the fission-like events, which was acknowledged to be most likely quasi-fission. Such times typically correspond to hundreds of rotations before scission, which would give mass distributions independent of angle.

Blocking measurements have also been carried out [19, 21] using beams of ${ }^{208} \mathrm{~Pb}$ and ${ }^{238} \mathrm{U}$ on crystalline targets of ${ }^{n a t} \mathrm{Ni}$ and ${ }^{n a t} \mathrm{Ge}$ at $\mathrm{E} / \mathrm{A}$ between 6.1 and $6.6 \mathrm{MeV}$. These reactions formed composite systems with $Z=114,120$ and 124. For $Z=114$, the blocking dip for heavier fission-like fragments was fitted with a lifetime of $1 \times 10^{-18} \mathrm{~s}$, but for lighter fragments the lifetime could only be defined as less than $10^{-18} \mathrm{~s}$. The blocking dips were more significantly filled for the reactions with ${ }^{238} \mathrm{U}$. For $\mathrm{Z}=120$, the measured blocking dip could be reasonably well reproduced with an exponential time distribution having a mean lifetime of $2.2 \mathrm{x}$ $10^{-18} \mathrm{~s}$. No exponential lifetime was quoted for $Z=124$, but the filling of the dip was similar, implying a similar result.

Despite the extraction of mean lifetimes corresponding to a single exponential time distribution, Ref. [19] gave a different physical interpretation to that of Refs. [18,20]. Morjean et al. concluded that their measured blocking dips indicate a fraction of long-lived fission $\left(>>10^{-18} \mathrm{~s}\right.$, thus having no blocking dip at all) of between $10 \%$ and $20 \%$ for the reactions forming $Z=120,124$. The long-lived fraction of events was associated with fusion-fission. Its presence was attributed to increasing fission barriers associated with superheavy element proton shell closure at Z 120,124. These conclusions may be tested through the extension of existing measurements of mass-energy distributions [23] to a much wider angular range.

\section{Comparison of results}

Comparison of crystal blocking time scales with the results from a correctly calibrated "neutron clock" and from measurements of MAD, for similar reactions, is an important tool to assess the consistency of the surprising results from the crystal blocking measurements.

As discussed in Sect. 3, MAD can give the most accurate characteristic times for reactions where less than a full rotation occurred. To compare with the blocking results, MAD were measured in Ref. [22] for the reactions of ${ }^{34} \mathrm{~S}+{ }^{186} \mathrm{~W},{ }^{48} \mathrm{Ti}+{ }^{186} \mathrm{~W}$ and ${ }^{64} \mathrm{Ni}+{ }^{184} \mathrm{~W}$ for a range of similar beam energies. These are shown in Fig. 8. For the ${ }^{32} \mathrm{~S}$ reaction, the grey shaded region around $\theta_{c . m .}=90^{\circ}$ shows where the detector geometry gave no coverage. The panels below show the $\mathrm{M}_{R}$ projections for $45^{\circ} \leq \theta_{c . m .} \leq 135^{\circ}$. They show marked differences between the three reactions. The ${ }^{64} \mathrm{Ni}$ reaction gives a minimum in yield at symmetry $\left(\mathrm{M}_{R}=0.5\right),{ }^{48} \mathrm{Ti}$ a broad peak around symmetry, whilst ${ }^{34} \mathrm{~S}$ gives a narrow peak. Together with the correlation of mass and angle seen in the MAD, quasi-fission with short average reaction times appears dominant in the ${ }^{64} \mathrm{Ni}$ and ${ }^{48} \mathrm{Ti}$ reactions.

To obtain quantitative reaction time scales for this dominant quasi-fission process, a classical Monte Carlo model was developed [22] to simulate quasi-fission MAD. The quasi-fission sticking time distributions were parameterized using a half Gaussian followed by an exponential decay. For simplicity it was assumed that the parameters are independent of angular momentum. The average and width of the Gaussian, and the decay time, were individually adjusted to reproduce the measurements. Using the time distributions shown in the top right panels of Fig. 8, the simulated MAD (shown below) reproduce the experimental 
EPJ Web of Conferences

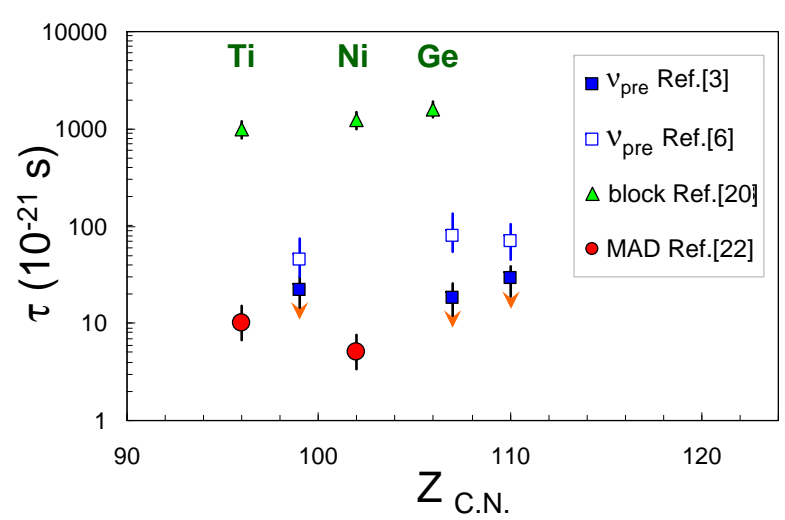

Fig. 9. Comparison of mean reaction times determined by different experimental methods, as a function of the compound nucleus atomic number $\mathrm{Z}_{C . N \text {. }}$.

MAD (left panels in Fig. 8) quite well. The $M_{R}$ spectra for $45^{\circ} \leq \theta_{c . m .} \leq 135^{\circ}$, shown in the lowest panels of Fig. 8, also agree with the experiment. Although similar MAD can be obtained by complementary adjustment of the peak time and decay time, the mean scission time for quasi-fission in the model is quite well defined for these reactions, at $5 \times 10^{-21} \mathrm{~s}$ for ${ }^{64} \mathrm{Ni}$, and $10 \times 10^{-21} \mathrm{~s}$ for ${ }^{48} \mathrm{Ti}$. These values are plotted in Fig. 9 as a function of the total charge of the system $\mathrm{Z}_{C . N .}$.

The pre-scission times from a simple three-source fit to neutron angular correlations for ${ }^{64} \mathrm{Ni}$-induced reactions were discussed in Sect. 2. Although a measurement for a $\mathrm{W}$ target was not made, time scales were determined for targets of ${ }^{154} \mathrm{Sm},{ }^{175} \mathrm{Lu},{ }^{197} \mathrm{Au}$ and ${ }^{208} \mathrm{~Pb}$. It is important to note that this analysis was carried out selecting masssymmetric fission-like events. These times are shown in Fig. 9 by filled blue squares. These represent upper limits, since the contributions from acceleration neutrons were not subtracted, thus downward-facing arrows are placed at the bottom of the error bars. Values are also shown from the analysis of Ref. [6] (open blue squares). That analysis did not account for measured neutron kinetic energies or contributions from acceleration neutrons (as discussed in Sect. 2) so over-estimates the times even more.

Finally the average lifetimes of Ref. [20], extracted from fits to blocking dips with an exponential time distribution, are shown in Fig. 9 by the triangles. The values plotted represent averages over the measured beam energies. For ${ }^{58} \mathrm{Ni}$ there was little variation with beam energy from $\mathrm{E} / \mathrm{A}=5.7$ to $6.5 \mathrm{MeV}$. The mean quasi-fission times extracted from MAD and the times determined by the "neutron clock" for mass-symmetric fission events agree quite well, when the effect of acceleration neutrons on the latter is recognised. The MAD measurements give no possibility that the mean lifetime of the quasi-fission process corresponds to more than a single rotation. The times for events close to masssymmetry in principle cannot be determined by the MAD, if they are assumed to originate from a different process to surrounding events. However the "neutron clock" measurements for ${ }^{64} \mathrm{Ni}$, taken at the same E/A as the highest energy ${ }^{58} \mathrm{Ni}$ blocking measurement, do not permit a long timescale even for the mass-symmetric events. Thus it must be concluded that the mean times from the blocking method, about 100 times longer than those extracted by the other methods, are inconsistent with expectations.

\section{Conclusions}

The reason for this timescale discrepancy is not obvious, as the blocking measurements have clearly been carried out with a great deal of care. It is desirable to resolve this problem, since the blocking method in principle provides a method to signal the presence of fusion-fission (and thus of fusion itself) within a large flux of quasi-fission.

Better understanding of the competition between fusion and quasi-fission, and thus of reaction mechanisms, should facilitate forming and investigating the properties of more super-heavy isotopes.

Having as many different measurements available for the same (or similar) reaction is clearly a desirable situation, which experimental research should pursue.

The extension of theoretical models to calculate and test their predictions simultaneously against all measured quantities will be the key test to determine which (or whether) existing approaches to modelling reaction dynamics in heavy element formation are fully correct.

\section{Acknowledgments}

The authors acknowledge financial support from ARC Grant DP110102858.

\section{References}

1. M.G. Itkis et al., Nuclear Physics A787(2007)150c

2. J. Tōke et al., Nuclear Physics A440(1985)327

3. D.J. Hinde et al., Phys. Rev. C 45(1992)1229

4. D.J. Hinde et al., Nuclear Physics A452(1986)550

5. D.J. Hinde et al., Nuclear Physics A553(1993)255c

6. K. Siwek-Wilczynska et al., Phys. Rev. C 51(1995)2054

7. J. Velkovska et al., Phys. Rev. C 59(1999)1506

8. D.J. Hinde et al., Phys. Rev. Lett. 52(1984)986; ibid. 53(1984)2275

9. H. Rossner et al., Phys. Rev. C 40(1989)2629

10. L. Donadille et al., Nuclear Physics A656(1999)259

11. T. Materna et al., Nuclear Physics A734(2004)184

12. W.Q. Shen et al., Phys. Rev. C 36(1987)115

13. R.G. Thomas et al., Phys. Rev. C 77(2008)034610

14. R. Rafiei et al., Phys. Rev. C 77(2008)024606

15. D.J. Hinde et al., Phys. Rev. Lett. 101(2008)092701

16. D.J. Hinde et al., Phys. Rev. Lett. 100(2008)202701

17. S.A. Karamian et al., Yad. Fiz. 14(1971)499

18. J.U. Andersen et al., Phys. Rev. Lett. 99(2007)162502

19. M. Morjean et al., Phys. Rev. Lett. 101(2008)072701

20. J.U. Andersen et al., Phys. Rev. C 78(2008)064609

21. M. Morjean et al., Eur. Phys. Jour. D45(2007)27

22. R. du Rietz et al., Phys. Rev. Lett. 106(2011)052701

23. E.M. Kozulin et al., Phys. Lett. B686(2010)227 